\title{
Identifying barriers in telesurgery by studying current team practices in robot-assisted surgery
}

\author{
Shirley A. Elprama, Katriina Kilpi, \\ Pieter Duysburgh, An Jacobs \\ iMinds-SMIT-Vrije Universiteit Brussel \\ Brussels, Belgium
}

\author{
Lotte Vermeulen, Jan van Looy \\ iMinds-MICT-Universiteit Gent \\ Gent, Belgium
}

\begin{abstract}
This paper investigates challenges in current practices in robot-assisted surgery. In addition, by using the method of proxy technology assessment, we provide insights into the current barriers to wider application of robot-assisted telesurgery, where the surgeon and console are physically remote from the patient and operating team. Research in this field has focused on the financial and technological constraints that limit such application; less has been done to clarify the complex dynamics of an operating team that traditionally works in close symbiosis. Results suggest that there are implications for working practices in transitioning from traditional robot-assisted surgery to remote robotic surgery that need to be addressed, such as possible communication problems which might have a negative impact on patient outcomes.
\end{abstract}

Keywords-component; robot-assisted surgery; telesurgery; da Vinci; surgical robot; robotically-assisted surgery; remote surgery; workflow; roles in the operating room.

\section{INTRODUCTION}

Robot-assisted surgery is increasingly becoming part of standard operating practice. Robot-assisted surgery allows surgeons to work on a patient indirectly in real-time, through a robotic controller. Doarn and Moses [1:71] define a surgical robot as "a powered, computer-controlled manipulator with artificial sensing that can be programmed to move and position tools to carry out a wide range of surgical tasks." More concretely, a surgical robot is a computer-assisted electromechanical device that is typically placed between a surgeon and patient during surgery. Surgeons sit at the console in the operating room outside the sterile area, where they control and manipulate the movement of one or more robot arms [2]. This allows for greater accuracy and precision than with traditional minimally invasive surgery, thanks to $3 \mathrm{D}$ visualization, six to seven degrees of freedom of manipulation and the potential of scaled movements, i.e., micromotion [3]. While many surgical robots have been developed over the years, only two robotic systems were made commercially available in clinical practice [1]: the Zeus platform (C) Computer Motion) (no longer currently available) and the da Vinci Surgical System (OIntuitive Surgical). Surgical robots are currently routinely used in minimally invasive abdominal surgery, pediatric surgery, gynecology, urology, cardiothoracic surgery, and otorhinolaryngology [2].
Although typically the controller of the robot (the 'master' side) occupies the same room as the instrumental side (the 'slave' side), robot-assisted surgery also offers the possibility for robot-assisted telesurgery. Since the distance between patient and surgeon is always greater in robot-assisted surgery than in open surgery or traditional minimally-invasive surgery, all forms of robot-assisted surgery can be understood as a remote robot-assisted surgery. However, in this paper we consider the term robot-assisted telesurgery as signifying any surgical intervention executed by a geographically distributed operating team, i.e. an operating team that is not located in the same physical operating room.

A breakthrough in remote robot-assisted surgery was made in 2001, when a patient in Strasbourg (France) was operated on by Dr. Marescaux in New York (USA) using the Zeus robot [4]. The first telesurgery using the da Vinci robot was done in 2005 by Colonel Noah Schenkman of the Walter Reed Army Medical Center (USA). This procedure was carried out on two pigs over the public Internet at the American Telemedicine Association event [5]. The U.S. Army's Telemedicine and Advanced Technology Research Center funded many of these subsequent experiments in collaboration with academia and industry.

\section{A. Thresholds for robot-assisted telesurgery}

Today robot-assisted telesurgery rarely takes place. To our knowledge and according to surgeons we spoke with, it does not take place in Flanders, Belgium. However, we were unable to retrieve exact numbers of occurrences of telesurgery in Flanders, Belgium to support this claim.

Nevertheless, theoretically robot-assisted telesurgery could be a way to bring the expertise of a surgeon to hospitals in rural areas or even to extreme locations, e.g. underwater stations or space stations [6]. Moreover, most test cases are simple and do not require complex tasks such as extensive dissection, suturing and knot tying [7]. This is due to a variety of thresholds that prevent the development and adoption of remote robot-assisted surgery on a large scale [1]. In general, we distinguish two types of thresholds: technical and nontechnical thresholds.

Technical thresholds, for instance, can refer to network latency issues [7]. For surgeons to perform well, there must be minimal degradation of the picture, minimal latency, high data 
quality and a robust communication system [1]. Furthermore, surgical robots currently lack tactile feedback, which omits an important source of information for surgeons during the operation [8]. However, this is partially compensated for by the possibility of seeing the video stream in $3 \mathrm{D}$.

Non - technical barriers include the high costs associated with telesurgery: "the cost of the robotic systems, the cost of telecommunication, the cost of personnel, the cost of infrastructure, the cost of training personnel and the cost of research and development" [1:91-92]. Next to these financial barriers, we also need to take into account jurisdictional differences across borders (e.g., differences in privacy legislation) and provide universal guidelines, licenses and/or laws [1].

However, less attention has been paid to the impact remote robot-assisted surgery has on the workflow of the operating team (OR team) [9]. Technical advances can have profound effects on workflow and may require new forms of cooperation. Some studies have already indicated that new technology changes the goals, tasks and responsibilities of the OR team [10][11]. Insights into these changes are of crucial importance since communication is frequently associated with medical accidents [12].

A geographically distributed OR team will have to collaborate in a new manner, finding new ways to communicate to bring the operation to a successful conclusion. In other words, next to the medical, financial and technical issues associated with the development of remote robotic surgery, the so-called social requirements [13] of the users of the surgical robots need to be taken into account as well.

Technological design must take into account peoples' work patterns within groups and organizations, in order to support the cooperative aspects of this work. It is therefore important to identify patterns of communication, roles and interactions between operating staff and incorporate these patterns into telesurgery systems. New technology in the operating room will undeniably cause changes in the workflow, and access to information [11]. As with the transition from minimal invasive surgery to robotic surgery, medical staff will have to deal with novel situations and new roles in robot-assisted telesurgery in remote locations. The technology is 'shaped' both by and for users: users adjust themselves and their surroundings to the new technology, and producers anticipate the use of the technology in its design by understanding current work practices [14]. A solid understanding of current practices of robotic surgery can help to identify the elements that could become challenging in geographically distributed OR teams. To accomplish this, we need to first understand how an OR team works today.

\section{B. The OR team}

An OR team consists usually of a surgeon, an anesthetist, a scrub nurse, and a circulating nurse. Depending on the type of intervention, this team can be extended with an assistant surgeon and multiple nurses. Apart from the OR team, other hospital staff also enters and leaves the operating room during the surgery. The operating room is not a closed environment: it stays relatively open for visits from colleagues. Also, some roles in the OR can be taken on by other members of the staff during individual interventions.

However, while members of the OR team have their own unique contribution, recent technological evolutions have shifted the surgeon away from the team to his own 'island'. An operating team working in a technologically complex operating room relies heavily on the surgeon, who is under increasing pressure to perform [15]. The surgical team cannot proceed without the surgeons, whose only communication medium is their voice and the actions of their tools, which the rest of the team can follow on screen. Next to the surgeon, the scrub nurse has a central role as the receiver, integrator and passer-on of information although this is often hampered by surgical masks that muffle speech [16]. Saunders [16] also suggests the use of reinforcing and confirming communication to make sure the message has been received as there are differences in how a message is interpreted.

Several authors have stressed the importance of collaboration within the OR team for successful and safe surgical care [17], [16], [18]. In fact, errors resulting from breakdowns in teamwork and communication in the OR can have catastrophic consequences for patients, families, caregivers, and entire institutions.

In this paper we will take a closer look at the interaction and communication patterns amongst the OR team in robotassisted surgery, and analyze these to understand what kind of interactions are crucial for a successful operation. By conducting this analysis, we contribute to the scarce amount of research on social and communication barriers in the context of robot-assisted surgery. Based on this analysis, we will identify implications for a geographically distributed operating team in robot-assisted telesurgery.

\section{METHOD}

\section{A. Proxy technology assessment (PTA)}

Although current technology enables the performance of robot-assisted telesurgery, such operations have, to our knowledge, only been executed exceptionally, and are not yet standardized clinical practice. Therefore, due to the limited number of medical professionals with first hand experience in carrying out telesurgery and the lack of telesurgeries to observe, we decided to study robot-assisted surgeries in a single room. We can then use the knowledge about these surgeries to conduct a proxy technology assessment for robotassisted telesurgery.

As such, this analysis can be understood as a 'proxy technology assessment' (PTA) [19]. PTA elaborates on the domestication theory [14], which states that new technology starts a process in which both the technology, the users and their social and physical surroundings undergo changes in which new practices can arise and old practices may be disrupted.

PTA is " a method for emulating everyday life practices with future technologies and applications by confronting selected user groups with existing similar tools and applications" [19:15]. The aim is to give users a future experience with one or more related technologies that are 
already available today. In this case, surgical robots for a geographically distributed OR team can be viewed as the new technology. In order to understand current practices in using surgical robots, we collected data by conducting seven interviews and observing 14 surgical procedures in various hospitals in Flanders, Belgium.

\section{B. Interviews}

The majority of the seven interviews were conducted during earlier stages of this research project. The main goal of these interviews was to gain more insight into the general practices during surgery and the roles of the people involved in minimal invasive surgery and robot-assisted surgery. In total, we interviewed two surgeons, two assistants, one scrub nurse, one biotechnician and IT support for the operating room across three different hospitals in Flanders, Belgium. All interviewees were involved in minimal invasive surgery, but none were experienced in robot-assisted surgery. However, since the interviews concentrated on general practices in surgery, the lack of experience in robot-assisted surgery did not make a difference.

\section{Observation}

We observed 14 surgical procedures (nine robot surgeries and five minimal invasive procedures) in four different hospitals in Flanders, Belgium. Since none of the authors of this paper had any prior knowledge of operating room practices, we observed both minimal invasive and robotassisted surgeries, in order to have a reference point for robotassisted surgeries and to be able to understand on which points they differed.

The minimal invasive procedures were done by surgeons specialized in either gastrointestinal surgery or general surgery and included procedures such as a gastric bypass and an explorative laparoscopy. All robot-assisted surgeries were done by surgeons specialized in urology and included procedures such as robot-assisted radical prostatectomy and robot-assisted partial nephrectomy. The robotic system used in all hospitals involved in robotic surgery, was the Da Vinci Surgical System from Intuitive Surgical.

\section{Forlizzi framework}

As a preparation for the observations, we created an observation protocol based on Forlizzi's product ecology framework [20]. This theory draws from social ecology and supports the investigation and description of how people build up social relationships with products by defining key factors in the ecology surrounding product use (i.e. products, people, roles, place) and how these change over time.

The observation protocol included themes such as personnel in the OR, the atmosphere in the OR, interactions amongst personnel, and other topics. When no further new occurrences were observed, we considered the observation to be complete.

\section{ANALYSIS}

During each observation, researchers made notes and took pictures. Also, for each operation, the researchers drafted a map of the configuration of the operating room (Figure 1). This data was combined and resulted in one observation report per visit to the hospital.

We used NVIVO software to code all observation reports. The first round of coding was mostly focused on identifying the interactions between surgical staff in the OR, while the second round was aimed at identifying current and future challenges related to robot-assisted telesurgery.

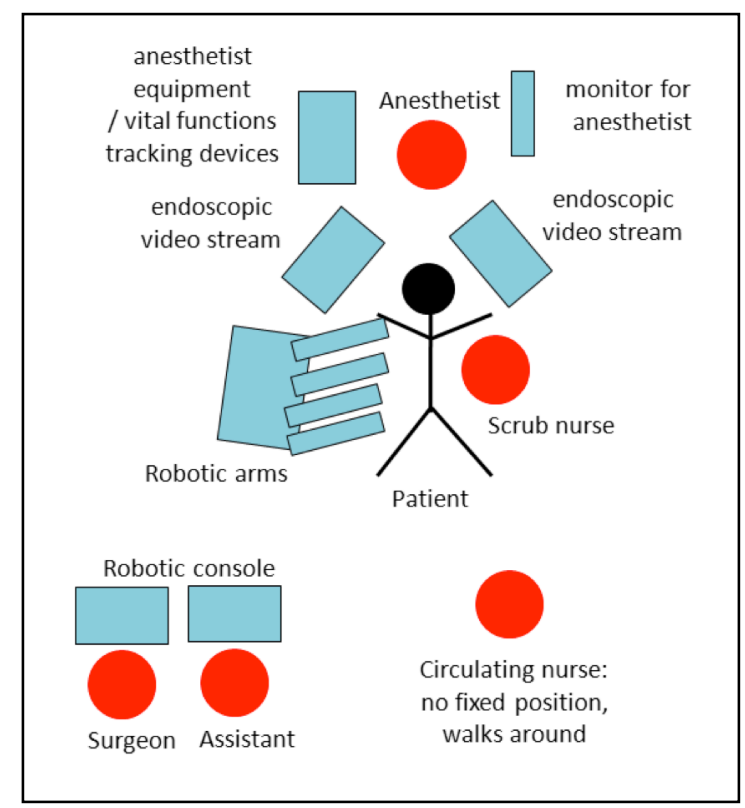

Figure 1. This schematic representation of an operating room during a robot-assisted surgery illustrates the distance between the surgeon and other staff in the OR.

\section{A. Mapping interactions of OR staff}

During the first round of coding, we selected text fragments from the robot-assisted surgery reports describing the actions of each member of the OR staff. We focused on surgical staff that had a main role in robot-assisted surgeries: surgeon, scrub nurse, assistant, circulating nurse and anesthetist. This exercise resulted in a long list of textual descriptions focusing mainly on actions and interactions of surgical staff in the OR. Interaction in this exercise meant any type of communication such as asking a question, agreeing with somebody, commenting, instructing or requesting.

When coding was complete, we mapped the interactions amongst the OR team. A poster visualizing the interactions was created for each member of the OR team. On this poster, we wrote the title of the OR staff member in the center and connected this with other people in the OR through arrows that detailed what type of interactions had been observed. As a result, we were able to visualize these interactions and their frequency amongst the OR personnel.

After looking at the frequencies and types of interactions more closely, we discussed how these would be affected if the OR team was to be geographically distributed. Based on these insights, a second coding iteration was done on the observational reports, this time concentrated on current and possible future challenges in robot-assisted surgery. 


\section{B. Identifying current challenges and future implications}

During the second iteration of coding, we re-read all the observation reports and focused on coding the current challenges and future implications in robot-assisted (tele)surgery. Current challenges refer to challenging or problematic situations as we observed in current robot-assisted surgery (e.g. OR staff having difficulties in hearing the surgeon when his voice is amplified through the microphone). Future implications refer to situations that might be problematic in surgeries that are remotely robot-assisted. As such, more insight in current and future challenges was gained. Also, while reflecting on these results and comparing them with literature, more challenges were identified.

\section{RESULTS}

As a result of this analysis, we have identified current challenges and future implications for robot-assisted (tele)surgery and listed them below. We start discussing each relevant topic with describing how the current situation is including present challenges. Then, we will explain what the implications are for the successful translation from robotassisted surgery to robot-assisted telesurgery with geographically distributed teams where the surgeon is not in the same space as the patient.

\section{A. Planning}

In order for a surgical procedure to take place, it is usually carefully planned, with the exception of urgent interventions. Surgeons, along with fellow surgeons from the medical department and the operating room manager, are normally in control of the planning.

Implication. In the context of remote surgeries, joint planning is required for both locations. The local OR needs to schedule the operation, and the remote surgeon and site of the console need to be included in this planning, which translates to an increase in the time required for planning and preparation.

\section{B. Preparing the procedure}

The procedure itself is discussed beforehand amongst the OR team, which includes the surgeon, the scrub nurse and the rest of the team. How this takes place differs between hospitals. For instance, at a particular hospital, a joint meeting was held at the start of the week where all operations of the coming week were discussed, but we also observed surgeons and their assistants discussing the procedure again before the start of the surgery (possibly a repetition of a previous meeting). Most of the interventions are standard interventions, and the team in the OR is usually aware of the preparations that are needed for a standard operation, for example, how the patient needs to be positioned, what material is needed, how the procedure will be executed and the type of support the operating surgeon requires during the intervention. When unusual, atypical interventions are to be carried out, the OR needs to consult more elaborately than usual.

Implications. Robot-assisted telesurgery is most likely of particular interest for highly specific interventions, since the surgeon is requested to conduct the operation for an atypical, rare type of intervention. As such, not only does this operation require more preparation due to the unusual nature of the intervention, but additional consultation is required between the remote surgeon and the local OR team in order for the local team to learn about the specific preferences of the surgeon with regard to the procedure.

A certain degree of freedom exists in which a surgeon can operate according to his or her personal preferences, such as specific tools. These preferences need to be provided and, where necessary, discussed as much as possible beforehand, in order not to unnecessarily prolong the duration of the operation. Other than the surgeon, scrub nurses might be wellplaced to help with these preparations, since they are already familiar with the preferences of 'their' surgeon.

\section{OR team size}

An enlarged OR team size means that the group that needs to be informed about the details of the procedure will necessarily be larger. In fact, there will always be a local assisting surgeon to carry out the surgical actions required to execute the robot surgery, for example, making incisions. Also, there needs to be a local surgeon in case of an incident that requires the robot surgery to be converted into open surgery (e.g. sudden bleeding), so this person and by consequence the whole OR team needs to be aware in detail of the procedure and when an intervention of his/her part will be required.

Implication. Since more medical staff is involved, more information needs to be shared with more people, thus increasing the chance of misunderstandings.

\section{Patient contact and trust}

Before surgery, surgeons usually have several consultations with their patients: surgeons visit patients before and after surgery, to inform them about the procedure and to reassure the patient.

Implication. While this task could be undertaken by the local (assisting) surgeon, or alternatively, the surgeon could contact the patient via teleconferencing tools, it is not certain if the patient would welcome such alternatives.

\section{E. OR status upon entry of surgeon}

In some of the operating rooms we observed, the surgeon entered the OR already at the start of the surgery, but in other ORs it was common that the surgeon arrives in the OR later than the rest of the OR team. By then, the nurses and the anesthetist have prepared the patient for surgery, and it is usually only after preparation that the surgeon is called to start the operation. Upon arrival at the OR, the surgeon typically takes some time to catch up on the current status of the patient. A surgeon can ask for changes in the setup of the OR (e.g. the type of light source, a different chair), before the operation starts. Only when the surgeon is up-to-date and the room is suitably set up are the first incisions made, if they have not been made by the assistant before the arrival of the surgeon.

Implication. Either the remote surgeon or their scrub nurses will have to communicate their preferences (see B.) in advance. The operating team on both sides might have to discuss the surgery beforehand and make sure everyone on both sides is prepared. Most likely, preparing for a robot-assisted telesurgery will take more time in the beginning but with routine, the preparation time will probably decrease. 


\section{F. Increased need for communication}

As mentioned before, an OR team is, generally speaking, used to working with each other. The ways of working and preferences of each staff member are known amongst the team. Also, a surgeon usually gives orders without explicitly mentioning to whom the order is addressed. The OR team is, in general, aware who the instructions are directed at without the surgeon having to explicitly mention. However, sometimes more explicit communication is needed. For instance, we observed the assistant surgeon waiting for confirmation from the head surgeon as to where to place a staple.

Implication. If the OR team is not familiar with the remote surgeon, it is to be expected that both sides will have to be more explicit about everything, since the team will be less able to anticipate the actions and preferences of the surgeon. Furthermore, each individual will have to be more alert than usual as the procedure in question is likely to be atypical.

\section{$G$. Language differences}

Surgery, like other fields, is increasingly international. During some of the surgeries we observed, foreign interns or assistant surgeons were present during the intervention. Particularly since robot-assisted surgery can still be largely regarded as a specialized type of medical intervention, the interns in the hospital are more likely to have an international profile as only some hospitals offer the possibility to become trained in robotic surgery. However, while English is a language most people in the OR team are able to speak, it is not their native language and communicating in a foreign language can at times be challenging, especially in situations of urgency. Problems can also occur when all OR team members have the same mother tongue: dialects might also cause misunderstandings.

Implications. Given the increased need for communication, being able to express oneself clearly is also of paramount importance. When the team is international, all members in the OR team will have to be able to speak the commonly agreed upon language fluently. Dialects can require some time to become accustomed to.

\section{H. The scrub nurse}

Similarly to open surgery and minimal invasive surgery, in a robotic surgery setting, the scrub nurse plays a pivotal role for the surgeon. Since the surgeon is located behind the robot console during surgery, the scrub nurse (or in some cases the assistant) is the person closest to the patient. Scrub nurses working in robotic surgery have usually followed additional training in order to be qualified for robotics and are highly specialized in this regard. There is often a very close relationship between the scrub nurse and surgeon. The surgeon frequently asks for the scrub nurse's opinion and respects his or her advice. Due to the fact that the surgeon and the scrub nurse work as a team, the scrub nurse is able to anticipate the surgeon's moves, and to suggest things they know to be the preference of the surgeon from experience. At the same time, the surgeon is able to trust that the scrub nurse will keep track of what is going on in the OR. Scrub nurses are the extra pair of ears and eyes and will alert the surgeon in case something worth noting occurs. It was also noted in the observations, that the surgeon was more attuned to the scrub nurse's voice than to those of the other members of the staff. This and the fact that it seemed that most things the surgeon said were addressed to the scrub nurse, underline the importance of the relationship between these two OR staff members.

Implication. Remote surgeons will probably have to work with a scrub nurse they are less familiar with, which will make the collaboration less smooth. It will probably take time to build up a similar working relationship in robot-assisted telesurgery.

\section{I. $\quad$ The circulating nurse}

The circulating nurse functions as an extra pair of hands and legs for the whole team but mostly for the surgeon and the scrub nurse. The scrub nurse remains in the sterile area, meaning that it is the task of the circulating nurse to deliver and remove all tools as directed by the scrub nurse. When the surgeon needs something (e.g. the light to be dimmed, a new chair to be brought in or a cup of coffee to be delivered), these requests will be addressed to the circulating nurse, either by the surgeon himself or by the scrub nurse who has already anticipated the need. Sometimes, the circulating nurse functions as a messenger between the surgeon and the scrub nurse. This is due to the humming of the robot, which occasionally prevents them from hearing what is being said in the OR clearly, despite the fact that what they say is amplified through the speaker system.

Implication. The surgeon in the remote location will have to have an assistant stand by to cater for the wishes that might arise. Along with the rest of the OR team, the circulating nurse will have to be familiar with the procedure and be able to anticipate the tools that will need to be delivered or removed. Most likely, the circulating nurse's interaction with the surgeon will diminish, as the circulating nurse will take orders from the scrub nurse or assisting surgeon located near the patient. The circulating nurse will in any case remain a crucial team member in the OR, guaranteeing a smooth and sanitary operation and therefore the patient's safety.

\section{J. Informal and non-verbal communication}

In minimally invasive surgery, the OR team is positioned closely together, enabling direct exchanges concerning matters both related and unrelated to surgery. In robot-assisted surgery, the surgeon is further away from the patient. Although in the latter case we observed less informal communication between the surgeon and the staff located nearest to the patient, some informal conversations still occurred between the surgeon and especially the scrub nurse (i.e. one informal discussion concerned work related issues). It is likely that such exchanges will be difficult for the surgeon, whose head is placed within the console and who must disengage his head in order to hear the others better, as we observed him do. If surgeons pull their head out from the console, they are consequently unable to continue with the surgery since they will no longer be able to visualize the operation.

When a visiting assistant was participating in the operation in the console next to the surgeon's (they were both behind a console), most communication was related to the operation. During instances where they were trying to communicate, they 
were both obliged to remove their head in order to hear each other properly and to be able to listen and speak properly.

However, the informal discussions around the patient were also fewer in number compared to minimal invasive surgery, most likely because the staff around the patient had to concentrate on looking at the screen to be able to follow the surgeon, and on the other hand, to listen carefully when the surgeon comments from the console through the microphone.

In contrast to minimal invasive surgery, where OR team members can also communicate using non-verbal language (e.g. nods, gesturing), non-verbal communication is impeded in robot-assisted surgery, with the surgeon sitting behind the robot console most of the time during local robot surgery.

Implication. Both informal and non-verbal interaction between the surgeon and the OR team are further altered by moving the surgeon further away from the rest of the OR team. When the surgeon is remote, interactions between the surgeon and the team will become less frequent, even though technology might support them to some extent (e.g. videoconferencing tools).

\section{$K$. Overview of what is happening in the $O R$}

Another challenge resulting from the increased distance between the surgeon and the rest of the OR team is the difficulty of having an overview of the OR. Since surgeons are sitting behind a console with their faces inside the console while looking inside the patient, it is difficult to see what is going on in the rest of the OR. Therefore, when there are problems with the robot (for example, when robot arms conflict with each other), surgeons usually do not immediately see the cause of the problem.

For instance, during one of the observations, the assistant was having problems connecting new instruments to the robot arms. Because this took a while, the surgeon stood up and walked to the assistant. After seeing the assistant struggling with the arms, the surgeon returned to the console. He then asked multiple times if he could continue surgery, while the assistant was still trying to fix the problem.

Also, the opposite happens. In some cases, OR staff such as the assistant had to wait for the surgeon, because he was texting or calling. Depending on his physical location in the OR, the assistant is unable to see what the surgeon is doing, or to have an understanding of what is going on. Also, sometimes the robot arms conflict with each other, and the scrub nurse has to reposition them to continue the operation.

Implication. Although all members of the OR team have individual tasks they are responsible for, the whole team needs to be aware of what is going on in the OR. That there is a need for the surgeon to approach the patient suggests that there is information lacking, which cannot be gained from the surgeon's position behind the console. This can become challenging when the surgeons find themselves in a remote location. For instance, the robot and the assistant should both be visible on screen to know what is going on closest to the patient.

\section{Having an overview of the operating area}

It is not only important to have an overview of what is happening inside the OR, but also paramount to have an overview inside the patient. For instance, during one of the minimal invasive procedures, we heard the surgeon saying: "Surgery is exposure: if you do not see well, you cannot do a decent job."

We observed multiple situations in which the surgeon 'lost' the assistant or the assisting scrub nurse. The surgeon is in control of the camera, which is attached to one of the robot arms, and also of all other robot arms. However, if the surgeon moves the camera too far or too rapidly away from the assistant's tools, the assistant can no longer see where his tools are. In case the surgeon loses their view of the assistant's tools, the surgeon has to move the camera and find them again.

Implication. In telesurgery, surgeons and assistants or scrub nurses need to a way to communicate that facilitates them easily finding each other.

\section{How different views can be difficult in collaborating together}

The next challenge is a technical one, but can lead to communication problems. When carrying out robot-assisted surgery, the surgeon, sitting behind the console that controls the robot arms, has a 3D view while the rest of the team watches a $2 \mathrm{D}$ representation on the other screens in the OR. The $3 \mathrm{D}$ view is necessary for the surgeon to compensate for the loss of tactile feedback. In the current situation, this can already cause confusion between the surgeon (who is in control of the camera, sees in 3D but has no tactile feedback) and the assistant (who sees in 2D but has tactile feedback).

This next fragment illustrates how the two different views can lead to misunderstandings. In this observed case, the surgeon wanted the assistant to place staples.

\section{Assistant: "Where do I place it?"}

Surgeon: "Wait, wait, wait. There." (the surgeon points at the place with his robotic grasper). The assistant follows his directions, but then the surgeon says "A little bit to the outside." The assistant moves the staple to one side when the surgeon comments: "That's to the inside."

In one of the ORs where we carried out an observation, however, there was a possibility to watch the video stream in the OR in 3D on a special 3D screen by using shutter glasses. However, according to the scrub nurse who assisted during that surgery, no one used these due to annoyance with the 3D glasses they had to wear, and due to the limited added value they offered.

Implication. Collaborating in robot-assisted surgery can be challenging due to the different views (2D vs. 3D). For remote robot-assisted surgery, this will probably remain an issue. To overcome these issues, a solution is needed that allows assistants to view the images in $3 \mathrm{D}$ as well. However, this solution should be seamlessly integrated into current working practices, as 3D glasses apparently do not offer a solution that fits user needs. 


\section{CONCLUSION AND DISCUSSION}

As described in the introduction, research on the barriers to establishing telesurgery in routine clinical practice has concentrated mainly on financial, legal or technological challenges (e.g. [1], [7]). However, in this paper, we focused on the social and communicational aspects of robot-assisted surgery, a topic that has gone largely unnoticed until recently (e.g. by [21]). Studying these aspects is important since communication errors may contribute to medical accidents [12]. By analyzing these challenges in the operating room environment during robot-assisted surgery, we aimed at identifying future implications for telesurgery using proxy technology assessment.

A number of future implications were identified, with varied levels of impact. For example, a flexible and capable surgical team will probably be able to adapt to a variety of conditions. However, together with the process of adaptation, more communication is needed, and adapting might result in a longer operation time. Indeed, both [12] and [21] found evidence that suggests more communication is needed.

In the study by [21], two surgical teams (French and American) conducting robot-assisted surgery were compared. It was found that that the less experienced team needed more time to prepare and had more verbal exchanges. Although it remains unclear to which degree this can be explained by cultural differences [21], similar evidence was found by Nyssen and Blavier [12]. Their work suggested that more communication is needed in robot-assisted surgery compared to minimal invasive surgery and was likely to be a sign of adaptation.

Although overcoming barriers requires adaption of the surgical team, there is also room for improvement on the technical side. For instance, the current speaker system of the Da Vinci surgical robot is far from ideal, as observed by the occurrence of miscommunication between surgeons and their team in our study. This underlines the importance of an adequate teleconferencing system. In the context of telesurgery, when the surgeon is at a remote location, all communication has to be mediated and therefore it becomes potentially more troubled.

To what extent will remote robot-assisted surgery ever become a reality? There are many issues that are highly problematic. Will remote robot-assisted surgery for instance ever be of use in an emergency, given the additional and more complex preparations that are foreseen? How will patients requiring a very specific type of expertise react to the notion of an operation where they are operated on from a significant distance? Will any kind of videoconferencing system allow the remote surgeon to come close to 'feeling' the local OR and support a collaboration that is as close as the collaboration that takes place within an OR team? And finally, will the setup become economically viable so that patients will also be able to recover from the costs of the procedure? Or will remote robotassisted surgery remain a thrill for the privileged few, much the same way space travel evolved in our recent past?

In the middle of all this is the surgeon, who has already been further removed from the rest of the OR team with the introduction of robot-assisted surgery. Robot surgery has increased the pressure on surgeons [15], allowing (but at the same also obligating) them to work with more than two instruments, executing interventions that previously could not be carried out via minimally invasive surgery. While this may have advantages for the patient, the pressure on the surgeon rises since the operations become more elaborate, requiring ever more advanced skills and knowledge. How this will influence the adoption of robot-assisted telesurgery remains to be seen. A further specialization in robot-assisted surgical skills could mean that these skills become even more scarce making remote surgery a more viable option.

Although we have identified a range of current and future challenges in robot-assisted surgery, we are not aware to which extent surgeons or other surgical staff would agree with the possible implications this has for telesurgery. The next step is presenting these challenges to people working in the OR such as the surgeon and the scrub nurse to know how real users of this new technology estimate the severity of these challenges. This will allow us to validate the findings as described in this paper and place the findings in the context of their professional working experience.

Proxy technology assessment method has its limitations: the real dynamics of a geographically distributed team cannot be fully predicted. However, in analyzing the use of current technology, a proxy technology assessment can make us aware of some of the issues that geographically distributed OR teams may be faced with. While telesurgery is not common, another suitable context in which the adaptation process of working with a new team could be studied in, is live surgical demonstrations. For instance, many surgeries are demonstrated at major surgical conferences [22]. In some cases, surgeons are invited to operate in a hospital and an operating room they are not familiar with. In their guidelines for surgical procedures, the authors advise surgeons to bring their own assistant, scrub nurse and important instruments with them [22]. This suggests that working with both familiar people and tools have their advantages and that the importance of studying this adaptation process should not go unnoticed.

Research covering classic issues related to computersupported communication (e.g. presence, privacy, trust) is highly relevant for the future development of telesurgery. For instance, Da Silva et al. [7] suggest that the development of telesurgery is dependent on various factors including acceptance by surgeons. Trust and the development of trust are likely to play an important role as well, given the surgeon who performs telesurgery has to put his trust in the skills (and possibly also the diagnosis) of the surgical team at the remote location.

\section{ACKNOWLEDGEMENTS}

We would like to thank the surgeons and hospital staff for allowing us in their operating rooms to observe. Also, we are thankful to the hospital staff that was willing to be interviewed. Finally, we thank Dervla Gleeson for proofreading various versions of this paper.

The Telesurgery project is co-funded by iMinds, a research institute founded by the Flemish Government, with project 
support of IWT. Companies and organizations involved in the project are Barco Healthcare Division and CandIT-Media BVBA.

\section{REFERENCES}

[1] C. R. Doarn and G. R. Moses, "Overcoming Barriers to Wider Adoption of Mobile Robotic Surgery: Engineering, Clinical and Business Challenges.," in Surgical Robotics - Systems, Applications, and Visions., J. Rosen, B. Hannaford, and R. Satava, Eds. 2011, pp. 69-102.

[2] D. M. Herron and M. Marohn, "A consensus document on robotic surgery," Surgical Endoscopy, vol. 22, no. 2, pp. 313-325, 2008.

[3] S. Bann, M. Khan, J. Hernandez, Y. Munz, K. Moorthy, V. Datta, T. Rockall, and A. Darzi, "Robotics in surgery.," Journal of the American College of Surgeons, vol. 196, no. 5, pp. 784-95, May 2003.

[4] J. Marescaux, J. Leroy, M. Gagner, F. Rubino, D. Mutter, M. Vix, S. E. Butner, and M. K. Smith, "Transatlantic robot-assisted telesurgery," Nature, vol. 413, no. 6854, pp. 379-380, 2001

[5] B. Challacombe, P. Dasgupta, U. Patel, P. Amoroso, and R. Kirby, "Recognizing and managing the complications of prostate biopsy," BJU international, vol. 108, no. 8, pp. 1233-1234, 2011.

[6] T. Haidegger, J. Sándor, and Z. Benyó, "Surgery in space: the future of robotic telesurgery," Surgical Endoscopy, vol. 25, no. 3, pp. 681690, 2011.

[7] V. da Silva, T. McGregor, R. Rayman, and P. P. W. Luke, "Telementoring and Telesurgery: Future or Fiction?," 2010.

[8] R. M. Satava, "Future Directions in Robotic Surgery," in Surgical Robotics: Systems Applications and Visions, J. Rosen, B. Hannaford, and R. M. Satava, Eds. Springer, 2010, p. 1.

[9] A. Blavier and A.-S. Nyssen, " $\{$ É $\}$ tude de l'impact des nouvelles technologies sur les modes de coop $\{$ é\}ration des chirurgiens par l'analyse des communications sur le terrain," Le travail humain, vol. 73, no. 2, pp. 123-140, 2010.

[10] D. Nio, W. a Bemelman, O. R. C. Busch, B. C. Vrouenraets, and D. J. Gouma, "Robot-assisted laparoscopic cholecystectomy versus conventional laparoscopic cholecystectomy: a comparative study.," Surgical endoscopy, vol. 18, no. 3, pp. 379-82, Mar. 2004.

[11] J. L. Webster and C. G. L. Cao, "Lowering Communication Barriers in Operating Room Technology," Human Factors: The Journal of the Human Factors and Ergonomics Society, vol. 48, no. 4, pp. 747758, Dec. 2006.
[12] A.-S. Nyssen and A. Blavier, "Communication as a sign of adaptation to a complex system: The case of robotic surgery," in Improving Healthcare Team Communication: Building on Lessons from Aviation and Aerospace, C. Nemeth, Ed. 2008, pp. 1-18.

[13] M. S. Ackerman, "The intellectual challenge of CSCW: The gap between social requirements and technical feasibility," Humancomputer interaction, vol. 15, no. 2, pp. 179-203, 2000.

[14] R. Silverstone, L. Haddon, R. In Silverstone, and R. Mansell, "Design and the domestication of \{ICTs:\} technical change and everyday life," Communicating by Design: The Politics of Information and Communication Technologies. Oxford: Oxford University Press. p44-74, 1996.

[15] S. Arora, L. Hull, N. Sevdalis, T. Tierney, D. Nestel, M. Woloshynowych, A. Darzi, and R. Kneebone, "Factors compromising safety in surgery: stressful events in the operating room," The American Journal of Surgery, vol. 199, no. 1, pp. 60-65, 2010.

[16] S. Saunders, "Why good communication skills are important for theatre nurses.," Nursing times, vol. 100, no. 14, pp. 42 - 44, 2004.

[17] S. Undre, A. N. Healey, A. Darzi, and C. A. Vincent, "Observational assessment of surgical teamwork: a feasibility study," World journal of surgery, vol. 30, no. 10, pp. 1774-1783, 2006

[18] J. B. Sexton, M. A. Makary, A. R. Tersigni, D. Pryor, A. Hendrich, E. J. Thomas, C. G. Holzmueller, A. P. Knight, Y. Wu, and P. J. Pronovost, "Teamwork in the operating room: frontline perspectives among hospitals and operating room personnel," Anesthesiology, vol. 105 , no. 5 , pp. $877-884$, Nov. 2006

[19] J. Pierson, A. Jacobs, K. Dreessen, B. Lievens, I. den Broeck, and W. den Broeck, "Walking the interface: uncovering practices through proxy technology assessment," Paper for EPIC, pp. 24-26, 2006.

[20] J. Forlizzi, "The product ecology: Understanding social product use and supporting design culture," Human-Computer Interaction Institute, p. 35, 2007.

[21] S. Cunningham, A. Chellali, I. Jaffre, J. Classe, and C. G. L. Cao, "Effects of experience and workplace culture in Human-Robot Team Interaction in Robotic Surgery: A Case Study," International Journal of Social Robotics, vol. 5, no. 1, pp. 75-88, 2013.

[22] B. Challacombe, R. Weston, G. Coughlin, D. Murphy, and P. Dasgupta, "Live surgical demonstrations in urology: valuable educational tool or putting patients at risk?," BJU International, vol. 106, no. 11, pp. 1571-1574, 2010. 